\title{
Expression of heregulin, phosphorylated HER-2, HER-3 and HER-4 in HER-2 negative breast cancers
}

\author{
SUSANNE HAAS ${ }^{1}$, HEIDRUN GEVENSLEBEN ${ }^{1}$, SYLVIA RABSTEIN ${ }^{2}$, VOLKER HARTH ${ }^{2}$, BEATE PESCH ${ }^{2}$, \\ THOMAS BRÜNING ${ }^{2}$, CHRISTINA JUSTENHOVEN ${ }^{3}$, HILTRUD BRAUCH $^{3}$, UTE HAMANN $^{4}$, \\ YON-DSCHUN KO ${ }^{5}$, CHRISTIAN BAISCH ${ }^{5}$, HANS-PETER FISCHER ${ }^{1}$ and REINHARD BÜTTNER ${ }^{1}$ \\ ${ }^{1}$ Institute of Pathology, Medical Faculty of the University of Bonn, Bonn; ${ }^{2}$ BGFA-Research Institute of Occupational \\ Medicine of the German Social Accident Insurance, Ruhr University Bochum, Bochum; ${ }^{3}$ Dr. Margarete Fischer-Bosch- \\ Institute of Clinical Pharmacology, Stuttgart, and University of Tübingen; ${ }^{4}$ Molecular Genetics of Breast Cancer, \\ Deutsches Krebsforschungszentrum (DKFZ), Heidelberg; ${ }^{5}$ Department of Internal Medicine, \\ Evangelische Kliniken Bonn gGmbH, Johanniter Krankenhaus, Bonn, Germany
}

Received July 31, 2008; Accepted October 14, 2008

DOI: $10.3892 /$ or_00000221

\begin{abstract}
A significant number of HER-2 amplified breast cancers is effectively treated by trastuzumab and further shows receptor-enhanced chemosensitivity. Recent studies have postulated transactivation of HER-2 also in tumors expressing phosphorylated/activated HER-2 (pHER-2) and of the HER-3/HER-4 ligand heregulin (HRG), independent of HER-2 amplification. As a consequence, a subset of tumors without HER-2 overexpression would be sensitive to trastuzumab chemotherapy. To investigate the potential transactivation of HER-2, in 171 breast cancers from the GENICA study with negative/low expression of HER-2 we analyzed the expression of pHER-2, HRG, HER-3 and HER-4 by immunohistochemistry. None of the tumors examined displayed expression of pHER-2. Moderate or strong cytoplasmic staining of HRG, HER-3 and HER-4 was observed in $44(26 \%), 67$ (39\%) and 33 (19\%) cases, respectively. No association of HRG, HER-3 and HER-4 with the survival of patients or with known prognostic clinical factors was seen. In conclusion, our data obtained on a well-characterized cohort of breast cancers provide no evidence of HER-2-activation in the absence of HER-2 overexpression. The biological function and clinical implications of HRG, HER-3 and HER-4 in this group of tumors remain unclear. Our results cannot support the hypothesis of a transactivation of HER-2 and thus a possible therapeutic benefit of trastuzumab in HER-2 negative breast cancers.
\end{abstract}

Correspondence to: Dr Susanne Haas, Institute of Pathology, Sigmund Freud Str. 25, D-53127 Bonn, Germany

E-mail: susanne.haas@ukb.uni-bonn.de

Key words: heregulin, HER-3, HER-4, immunohistochemistry

\section{Introduction}

Overexpression and/or amplification of the receptor tyrosine kinase HER-2 occurs in 15-20\% of breast cancers and is related to a shorter overall survival (1). A significant number of these tumors is effectively treated by trastuzumab, an antibody directed to the ectodomain of p185 ${ }^{\mathrm{HER}-2}$.

In breast cancer cells with HER-2 amplification, HER-2 is phosphorylated by spontaneous homodimerization. Another generally accepted mechanism of HER-2 activation takes place by ligand-mediated heterodimerization with other members of the human epidermal growth factor receptor family, EGFR-1, HER-3 or HER-4 (2). Heregulin (HRG, also called neuregulin 1, NRG 1), is a ligand for HER-3 and HER-4, leading to transphosphorylation and transactivation of HER-2 $(2,3)$. In vitro studies have demonstrated a stimulating effect on proliferation in breast cancer cells by low concentrations of HRG, regardless of HER-2 overexpression. High concentrations of HRG on the other hand result in growth inhibition in cells overexpressing HER-2 (4,5). Further observations have indicated that overexpression of HRG induces a more aggressive, hormone-independent phenotype in breast cancer cells that do not overexpress any of the HER receptors (6). Nonetheless blockage of HRG expression in breast cancer cell lines inhibits cell proliferation and thus the malignant metastatic potential of the tumor cells in vitro (7).

These results indicate a possible therapeutic benefit from blocking HRG in breast cancers. Binding of HRG and the stimulating effect on cell cycle progression is blocked by monoclonal antibodies to HER-2 in breast and ovarian tumor cells, but this effect depends on expression of HER-2 in the tumor cells (2). However, Menendez et al have provided evidence that trastuzumab inhibits HRG induced HER-2/-3 phosphorylation and enhances chemotherapeutic effects of cisplatin even in breast cancer cells with weak or negative HER-2 expression but high levels of HRG and phosphorylated/activated HER-2 (8). The authors postulate that this subgroup of tumors would benefit from chemotherapy with trastuzumab and/or cisplatin/ taxan even in the absence of HER-2 overexpression. 


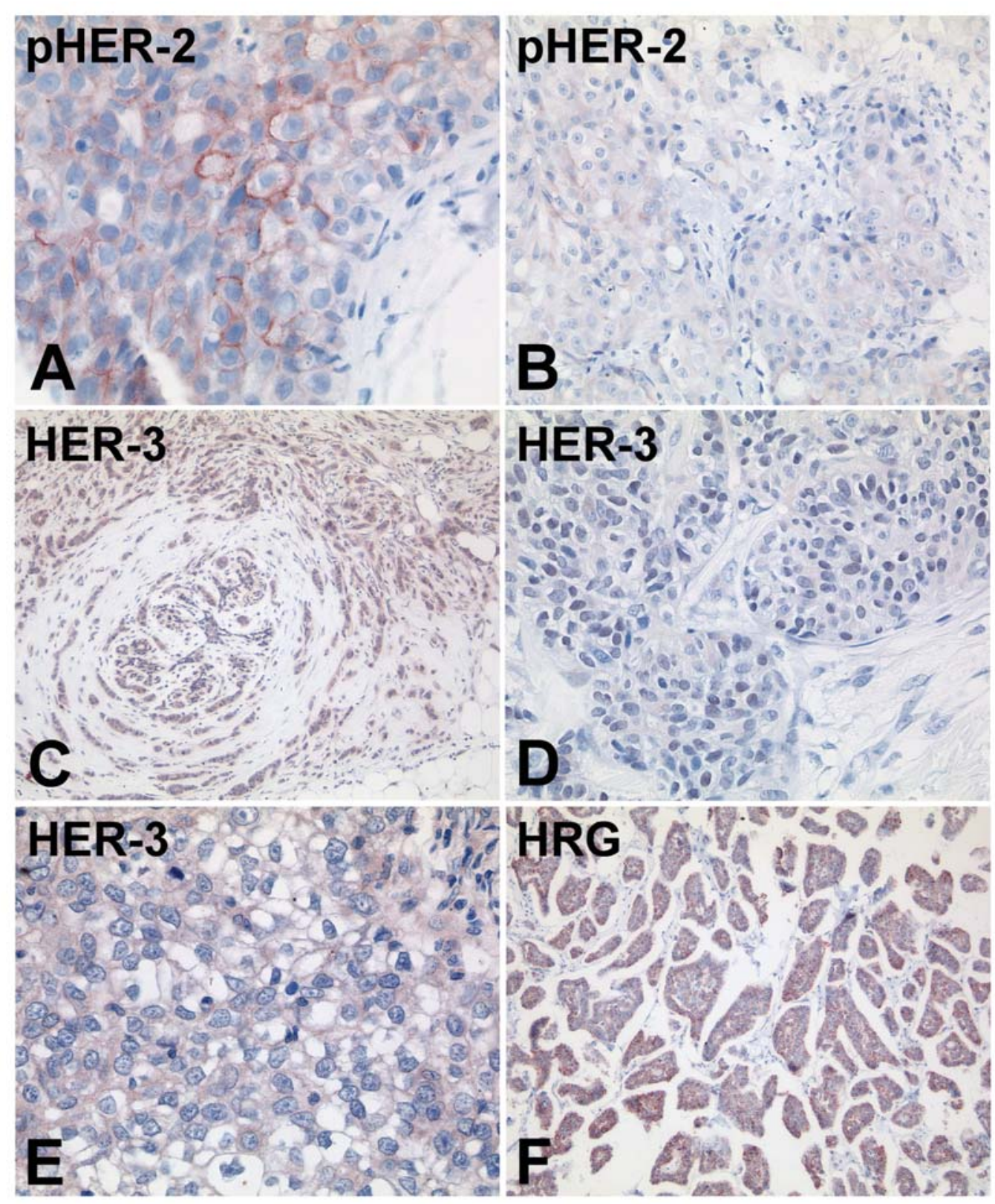

Figure 1. Immunohistochemical evaluation of pHER-2 revealed distinct membrane staining in HER-2 positive controls and focally within the HER-2 positive in situ component (A) of an otherwise HER-2 negative invasive ductal carcinoma (B). HER-3 staining was mostly cytoplasmic within the tumor cells, but also in non-neoplastic breast tissue (C). A few cases displayed faint nuclear or membrane staining of HER-3 (D and E). HRG staining of tumor cells was always cytoplasmic (F).

Considering the crucial therapeutic consequences resulting from these oberservations we investigated the expression levels of HRG with phosphorylated HER-2 (pHER-2), HER-3 and HER-4 in HER-2 negative human breast cancers of the GENICA population-based breast cancer casecontrol collection.

\section{Materials and methods}

Patients. Incident breast cancer patients and tumor tissues were recruited from the GENICA study (Interdisciplinary Study Group on Gene-Environment Interaction and Breast Cancer in Germany) that was conducted as population-based case-control study during 2000-2004. Inclusion criteria for study subjects were a histopathologically confirmed diagnosis of first primary breast cancer, Caucasian descent, residency in the greater Bonn area in Germany and age $<80$ years. The ethics committee of the University of Bonn approved the GENICA study. All participants gave written informed consent. Tissue samples from 171 patients with negative HER-2 status were available at Bonn University for immunohistochemical analysis.

Immunohistochemistry. Tissue specimens were fixed in $4 \%$ buffered formaldehyde and embedded in paraffin. Immunohistochemical stainings were performed on 4- $\mu \mathrm{m}$ sections with an immunostainer (Techmate 500; Dako Glostrup, Denmark). Citrate buffer $(0.1 \mathrm{M})$ was used as unmasking 
Table I. Immunohistochemical expression of heregulin (HRG), HER-3 and HER-4 in HER-2 negative breast cancers.

\begin{tabular}{lccccc}
\hline & $\begin{array}{c}\text { Number of } \\
\text { patients }\end{array}$ & $\begin{array}{c}\text { HRG } \\
\text { positive }\end{array}$ & $\begin{array}{c}\text { HER-3 } \\
\text { positive }\end{array}$ & $\begin{array}{c}\text { HER-4 } \\
\text { positive }^{\mathrm{a}}\end{array}$ & $\begin{array}{c}\text { HRG } \\
\text { negative }^{\mathrm{b}}\end{array}$ \\
\hline $\mathrm{N}$ & $171(\%)$ & $44(\%)$ & $67(\%)$ & $33(\%)$ & $100(\%)$ \\
HER-2 Score 0 & $78(45)$ & $22(50)$ & $26(39)$ & $14(42)$ & $44(44)$ \\
HER-2 Score 1+ & $93(54)$ & $22(50)$ & $41(61)$ & $19(57)$ & $56(56)$ \\
HRG positive & $44(26)$ & -- & $14(21)$ & $12(36)$ & -- \\
HER-3 positive & $67(39)$ & $14(32)$ & -- & $18(55)$ & $39(39)$ \\
& & & & p=0.044 & $11(11)$ \\
HER-4 positive & $33(19)$ & $12(27)$ & $18(27)$ & -- & $93(93)$ \\
ER positive & $157(91)$ & $41(93)$ & $61(91)$ & $32(97)$ & $7(7)$ \\
ER negative & $14(8)$ & $3(7)$ & $6(9)$ & $1(3)$ & $83(83)$ \\
PR positive & $142(83)$ & $37(84)$ & $54(81)$ & $29(88)$ & $17(17)$ \\
PR negative & $29(17)$ & $7(16)$ & $13(19)$ & $4(12)$ & \\
\hline
\end{tabular}

${ }^{\text {a Score }} \geq 40 ;{ }^{\text {b }}$ Score 0 .

solution ( $\mathrm{pH} 6.0$ for HRG, HER-3, HER-4; pH 9.0 for pHER-2). The antigen-antibody binding was visualized by means of the avidin-biotin complex (ABC-method) using AEC (3-amino-9-ethylcarbazol) as chromogen.

The following primary antibodies were used: HRG mouse monoclonal antibody, clone P137 (Novacastra, Newcastle upon Tyne, UK), HER2-pY-1248 (pHER-2) mouse monoclonal antibody, clone PN2A and HER-2 mouse monoclonal antibody, clone A0485 (Dako).

HER-3 mouse monoclonal antibody, clone SGP1, and HER-4 mouse monoclonal antibody, clone HFR-1 (Labvision, Fremont CA, USA). All antibodies were incubated overnight at $4^{\circ} \mathrm{C}$. Dilutions were 1:25 for HRG, HER2-pY and HER-4, 1:50 for HER-3.

Evaluation of immunohistochemistry. For HER-2 staining, the Hercep Test scoring method was used (Dako), considering only complete membrane staining in $>10 \%$ of cells to be positive (Dako Score $2+, 3+$ ). All tumors in this study showed absent or incomplete membrane staining for HER-2 (Dako score $0,1+$ ). For pHER-2, only membrane staining was considered positive.

Scoring of cytoplasmic staining of HER-3, HER-4 and HRG was performed as follows: the percentage of cells stained $(0-100 \%)$ was multiplied with the staining intensity (1, weak; 2 , moderate and 3 , strong) to give a maximum histoscore of 300. Tumors were considered positive for cytoplasmic staining if the score was 40 and higher.

If present, any nuclear staining of HER-3 and HER-4 was assessed positive. Estrogen and progesterone receptor positive tumors showed positive nuclear staining in at least $10 \%$ of the tumor cells. For statistical analysis the $\chi^{2}$ test was used.

\section{Results}

Immunohistochemical staining of pHER-2, HER-3, HER-4 and $H R G$ in breast cancer tissue. Distinct membrane staining of pHER-2 was seen in a group of HER-2 overexpressing breast cancers that served as positive controls and furthermore within the HER-2 positive in situ component of otherwise HER-2 negative invasive carcinoma (Fig. 1A and B). We failed to detect any membrane pHER-2 staining in any of the tumors lacking HER-2 overexpression. Five tumors displayed faint cytoplasmic staining for pHER-2 which was not considered positive and did not correlate with HER-2 status.

Weak to moderate cytoplasmic staining of $\mathrm{HRG}$ was a constant finding in non-neoplastic mammary glands, fibroblasts and vascular smooth muscle cells. Staining within the tumor cells also turned out to be cytoplasmic (Fig. 1F). Nuclear or membrane staining for HRG was not observed.

HER-3 staining was occasionally seen in the nonneoplastic breast tissue. Expression within tumor cells was mainly cytoplasmic. Only a few cases showed faint nuclear $(n=9)$ or membrane $(n=6)$ staining (Fig. 1C-E). Staining of HER-4 was similar with only a few cases revealing nuclear or membrane staining. The results of cytoplasmic staining of HER-3 and HER-4 are summarized in Tables I-III.

Coexpression of the HER proteins, estrogen and progesterone receptors, and HRG. There was no difference between tumors without HER-2 expression and those demonstrating weak incomplete membrane staining (assessed as hercep score 1+) referring to expression of pHER-2, HER-3, HER-4 and HRG. HRG was expressed in $26 \%$ of the tumors. No significant coexpression of HRG with HER-3 or HER-4 was observed. Significant coexpression of HER-4 positive 
Table II. Correlation of immunohistochemical HRG-, HER-3- and HER-4 expression with tumor chracteristics of HER-2 negative breast cancers.

\begin{tabular}{lrrrrr}
\hline & Number of patients & HRG positive & HER-3 positive & HER-4 positive & HRG negative $^{\mathrm{b}}$ \\
\hline $\mathrm{N}$ & $171(\%)$ & $44(\%)$ & $67(\%)$ & $33(\%)$ & $100(\%)$ \\
$\mathrm{T} 1$ & $104(61)$ & $23(52)$ & $42(63)$ & $20(60)$ & $65(65)$ \\
$\mathrm{T} 2$ & $50(29)$ & $14(32)$ & $19(28)$ & $10(30)$ & $28(28)$ \\
$\mathrm{T} 3$ & $11(6)$ & $4(9)$ & $2(3)$ & 0 & $5(5)$ \\
T4 & $6(4)$ & $3(7)$ & $4(6)$ & $3(9)$ & $2(2)$ \\
N0 & $105(61)$ & $24(55)$ & $38(57)$ & $18(55)$ & $65(65)$ \\
N1-3 & $66(39)$ & $20(46)$ & $5(43)$ & $15(45)$ & $35(35)$ \\
G1 & $13(8)$ & $2(5)$ & $37(55)$ & $15(4)$ & $8(8)$ \\
G2 & $84(49)$ & $18(41)$ & $25(37)$ & $15(45)$ & $52(52)$ \\
G3 & $74(43)$ & $24(55)$ & & & $40(40)$ \\
\hline
\end{tabular}

${ }^{\text {aS }}$ core $\geq 40$; ${ }^{\text {bScore } 0 .}$

Table III. Correlation of immunohistochemical HRG-, HER-3- and HER-4 expression and other factors with overall and disease-free survival in HER-2 negative breast cancers.

\begin{tabular}{lccccc}
\hline & Number of patients & OS $<5$ years & OS $>5$ years & DFS $<5$ years & DFS $>5$ years \\
\hline $\mathrm{N}$ & $88(\%)$ & $9(\%)$ & $79(\%)$ & $22(\%)$ & $66(\%)$ \\
HER2 Score 0 & $42(48)$ & $5(56)$ & $37(47)$ & $11(50)$ & $31(47)$ \\
HER2 Score 1 & $46(52)$ & $4(44)$ & $42(53)$ & $11(50)$ & $35(53)$ \\
HRG positive & $20(23)$ & $3(33)$ & $17(21)$ & $6(27)$ & $14(21)$ \\
HER-3 positive & $42(48)$ & $6(67)$ & $36(45)$ & $12(55)$ & $30(45)$ \\
HER-4 positive & $20(23)$ & $1(11)$ & $19(24)$ & $5(23)$ & $15(23)$ \\
ER positive & $79(90)$ & $9(100)$ & $70(89)$ & $19(86)$ & $60(91)$ \\
ER negative & $9(10)$ & 0 & $9(11)$ & $3(14)$ & $6(9)$ \\
PR positive & $68(77)$ & $9(100)$ & $59(75)$ & $17(77)$ & $51(77)$ \\
PR negative & $20(23)$ & 0 & $20(25)$ & $5(23)$ & $15(23)$ \\
T1 & $56(64)$ & $3(33)$ & $53(67)$ & $13(59)$ & $43(65)$ \\
T2-4 & $32(36)$ & $6(66)$ & $26(33)$ & $9(41)$ & $23(35)$ \\
N0 & & & $\mathrm{p}=0.047$ & & $42(64)$ \\
N1-3 & $89(56)$ & 0 & $49(62)$ & $7(32)$ & $24(36)$ \\
G1 & $39(44)$ & $9(100)$ & $30(38)$ & $15(68)$ & $\mathrm{p}=0.009$ \\
G2-3 & $80(91)$ & $9(100)$ & $71(90)$ & $22(100)$ & $5(11)$ \\
\hline
\end{tabular}

OS, overall survival; DS, disease-free survival.

tumors with HER-3 was detected: $55 \%$ of HER-4 positve tumors were HER-3 positive, $\mathrm{p}=0.0441$. This did not apply to HER-3 in the reverse case.

A minority of tumors was negative for the estrogen receptor $(\mathrm{ER}, 8 \%)$ or progesterone receptor $(\mathrm{PR}, 17 \%)$. No significant association of ER or PR with expression of HRG, HER-3 or HER-4 was observed. Results are given in Table I.

Correlation of immunohistochemical results with tumor characteristics and clinical follow-up. As demonstrated in 
Table II no significant associations of immunohistochemical expression levels with tumor size, grading or nodal status were detected.

Follow-up data were available in 88 cases (Table III). Nine patients died and 22 patients suffered from recurring disease within five years after diagnosis. The occurrence of lymph node metastasis correlated significantly with shortened disease-free survival $(\mathrm{p}=0.009)$ and overall survival $(\mathrm{p}<0.001)$. Advanced T-stages $(\mathrm{T} 2-4)$ correlated with shortened overall survival $(\mathrm{p}=0.047)$. However, no correlation of overall and/or disease-free survival with HRG, HER-3 and HER-4 expression was seen.

\section{Discussion}

Regarding new therapeutic options for the treatment with HER-2 antagonists, it is of special interest whether there is any evidence of transactivation of HER-2 in breast cancers without HER-2 overexpression. In our study, we therefore focused on HER-2 negative breast cancers to investigate expression levels of HRG, phosphorylated HER-2 (pHER-2), HER-3 and HER-4.

Our first important finding was the complete lack of pHER-2 in our study group. This result confirms a previous study from Thor et al who failed to detect pHER-2 in 509 breast cancer cases without HER-2 expression (9). In addition, we found that even tumors with low expression of HER-2 did not express pHER-2. Obviously, pHER-2 expression is restricted to tumors with high overexpression/amplification of HER-2. These findings are in contrast to a study by Menendez et al (8). These authors investigated pHER-2 expression in 189 breast cancers including 60 HER-2 positive tumors. Phosphorylated HER-2 was expressed in $45 / 189$ breast cancer cases, but only 7 of these 45 cases were HER-2 positive. From this it follows that 38 of 129 HER-2 negative cases (29\%) expressed pHER-2. In contrast, our data do not confirm these results. Of note, the present study as well as the studies by Menendez et al and Thor et al obviously were using the same primary antibody fo detection of tyrosine phosphorylated erbB2 (PN2A) $(9,10)$. An explanation may be differences in the case cohorts with regard to the age at diagnosis, histopathological para-meters and ethnic group.

HRG, a ligand of HER-3 and HER-4, is being considered a keyfactor for HER-2 transactivation. Several studies have demonstrated expression of HRG in breast cancer tissue (8,11-13). Although different primary antibodies and different methods of staining assessment were used, cytoplasmic expression of HRG in 30-50\% of breast cancers was a corresponding finding of all studies. We also found positive expression of HRG in $24 \%$ of the tumors, using a scoring level of $40 / 300$ as cut-off for positive expression. There are conflicting results on possible implications of HRG for tumor biology. A more aggressive phenotype of breast cancer cell lines expressing HRG has been described earlier $(5,6)$. On the other hand, authors have reported a poorer prognosis for tumors with low expression levels of HRG (11). We could not identify any correlation of absent expression or overexpression of HRG with clinical tumor data and survival.
In addition, we have no evidence of HER-2 activation/ phosphorylation in HER-2 negative tumors that overexpress HRG. This is in contrast to the results of Menendez et al who found $67 \%$ of HRG-overexpressing tumors to be in active (phosphorylated) HER-2 status (8). Furthermore, we found no relationship of HRG expression with expression of its receptors HER-3 and HER-4. Based on our immunohistochemical data, the role of HRG in tumors without HER-2 overexpression and without HER-2 phosphorylation is unclear.

Independent of HER-2, both HER-3 and HER-4 may become activated by binding of different ligands and homodimerization (14). Expression of HER-4 could be demonstrated in several types of human tissues and tumors $(15,16)$. Cleavage of the HER-4 protein leads to translocation of the intracellular domain into the nucleus and regulation of gene transcription (17). Therefore, membrane, cytosolic or nuclear isoforms of HER-4 may be detected by immunohistochemistry due to the different primary antibodies. The HFR-1 antibody used in the present study recognizes the intracellular domain of HER4, thus showing preferentially cytoplasmatic and rarely nuclear staining (18). Tovey et al assumed a protective effect of HER-4 if homodimerization occurs, but loss of this effect when other HER members were activated by heterodimerization with HER-4 (18). We were able to confirm coexpression of HER-4 with HER-3, as reported previously (19). However, neither of them was associated with clinical outcome or known prognostic factors in our breast cancer group.

In conclusion, we have no evidence of HER-2 activation/ phosphorylation in HER-2 negative tumors based on immunohistochemical data. Although we found HRG expressed in a substantial number of tumors, the biological function of HRG as well as its receptors HER-3 and HER-4 in absence of HER-2 activation remains to be elucidated.

\section{Acknowledgements}

We are indebted to all women participating in the GENICA study and we gratefully acknowledge support by interviewers. This work was supported by the Federal Ministry of Education and Research (BMBF) Germany grants 01KW9975/5, 01KW9976/8, 01KW9977/0, and 01KW0114 and by F. Hoffmann-La Roche Ltd.

\section{References}

1. Slamon DJ, Godolphin W, Jones LA, Holt LA, Wong SG, Keith DE, Levin WJ, Stuart SG, Udove J, Ullrich A, et al: Studies of the Her-2 proto-oncogene in human breast and ovarian cancer. Science 244: 707-712, 1989.

2. Lewis GD, Lofgren JA, McMurtrey AE, Nujens A, Fendly BM, Bauer KD and Sliwkowski MX: Growth regulation of human breast and ovarian cancer cells by heregulin: evidence for the requirement of ErbB2 as a critical component in mediating heregulin responsiveness. Cancer Res 56: 1457-1465, 1996.

3. Earp HS, Dawson TL, Li X and Yu H: Heterodimerizytion and functional interaction between EGF receptor family members: a new signalling paradigm with implications for breast cancer research. Breast Cancer Res Treat 35: 115-132, 1995.

4. Lupu R, Cardillo M, Harris L, Hijyzi M and Rosenberg K: Interaction between erbB-receptors and heregulin in breast cancer tumor progression and drug resistance. (Review) Semin Cancer Biol 6: 135-145, 1995.

5. Lupu R, Cardillo M, Cho C, Harris L, Hijazi M, Perez C, Rosenberg K, Yang D and Tang D: The significance of heregulin in breast cancer tumor progression and drug resistance. Breast Cancer Res Treat 38: 57-66, 1996. 
6. Tang CK, Perez C, Grunt T, Waibel C, Cho C and Lupu R: Involvement of heregulin-beta2 in the acquisition of the hormone-independent phenotype of breast cancer cells. Cancer Res 56: 3350-3358, 1996

7. Tsai MS, Shamon-Taylor LA, Mehmi I, Tang CK and Lupu R: Blockage of heregulin expression inhibits tumorigenicity and metastasis of breast cancer. Oncogene 22: 761-768, 2003.

8. Menendez JA, Mehmi I and Lupu R: Trastuzumab in combination with heregulin-activated Her-2 (erbB-2) triggers a receptor-enhanced chemosensitivity effect in the absence of Her-2 overexpression. J Clin Oncol 24: 3735-3746, 2006.

9. Thor AD, Liu S, Edgerton S, Moore D, Kasowitz KM, Benz CC, Stern DF and DiGiovanna MP: Activation (tyrosine phosphorylation) of ErbB-2 (HER2/neu): a study of incidence and correlation with outcome in breast cancer. J Clin Oncol 18: 3230-3239, 2000.

10. DiGiovanna MP and Stern DF: Activation state-specific monoclonal antibody detects tyrosine phosphorylated p185neu/ erbB-2 in a subset of human breast tumors overexpressing this receptor. Cancer Res 55: 1946-1955, 1995.

11. Raj EH, Skinner A, Mahij U, Nirmala KN, Ravichandran K, Shanta V, Hurst HC, Gullick WJ and Rajkumar T: Neuregulin 1 -alpha expression in locally advanced breast cancer. Breast 10 : 41-45, 2001.

12. Dunn M, Sinha P, Campbell R, Blackburn E, Levinson N, Rampaul R, Bates T, Humphreys S and Gullick WJ: Coexpression of neuregulins 1, 2, 3, 4 in human breast cancer. J Pathol 203: $672-680,2004$

13. Esteva FJ, Hortobagyi GN, Sahin AA, Smith TL, Chin DM, Liang SY, Pusztai L, Buzdar AU and Bacus SS: Expression of erbB/HER receptors, heregulin and P38 in primary breast cancer using quantitative immunohistochemistry. Pathol Oncol Res 7: 171-177, 2001.
14. Revillion F, Lhotellier V, Hornez L, Bonneterre J and Peyrat JP: ErbB/HER ligands in human breast cancer, and relationships with their receptors, the bio-pathological features and prognosis. Ann Oncol 19: 73-80, 2008

15. Srinivasan R, Poulsom R, Hurst HC and Gullick WJ: Expression of the c-erbB-4/HER4-protein and mRNA in normal human adult and fetal tissues and in a survey of nine solid tumor types. J Pathol 185: 236-245, 1998.

16. Prigent SA, Lemoine NR, Hughes CM, Plowman GD, Selden C and Gullick WJ: Expression of the c-erbB3 protein in normal human adult and fetal tissues. Oncogene 7: 1273-1278, 1992.

17. Junttila TT, Sundvall M, Lundin M, Lundin J, Tanner M, Härkönen P, Joensuu H, Isola J and Elenius K: Cleavable ErbB4 isoform in estrogen receptor-regulated growth of breast cancer cells. Cancer Res 65: 1384-1393, 2005.

18. Tovey MS, Dunne B, Witton CJ, Cooke TG and Bartlett JMS: HER4 in breast cancer: comparison of antibodies against intraand extra-cellular domains of HER4. Breast Cancer Res 8: R19, 2006.

19. Wiseman SM, Makretsov N, Nielsen TO, Gilks B, Yorida E, Cheang M, Turbin D, Gelmon K and Huntsman DG: Coexpression of type 1 growth factor receptor family members HER-1, HER-2, and HER-3 has a synergistic negative prognostic effect of breast carcinoma survival. Cancer 103: 1770-1777, 2005. 